\title{
"Edhin Krokowski aus Linde bei Pinne, Provinz Posen." Judentum und Antisemitismus im Zauberberg und seiner Vorgeschichte
}

\author{
YAHYA ELSAGHE \\ Universität Bern
}

Auf die Frage, ob und wie gegebenenfalls jüdische Figuren in ihnen vorkommen, geben Thomas Manns Erzähltexte ganz verschiedene Antworten. Diese variieren einerseits je nach dem historischen Moment, zu dem die entsprechenden Texte entstanden, andererseits aber auch nach den persönlichen Umständen, in denen sich der Autor zur je gegebenen Zeit befand. Das zeigt sich schon nur bei einer flüchtigen Sichtung des Erzählwerks, aus dem man zu diesem Zweck ein Frühwerk isolieren und gegen die späteren und spätesten Texte differenzieren kann. Das Frühwerk darf man etwa 1905 enden lassen: rezeptionsgeschichtlich mit dem zeitlich leicht verzögerten Großerfolg der Buddenbrooks; lebensgeschichtlich mit der so ermöglichten oder erleichterten Einheirat ins jüdische Großbürgertum; literaturgeschichtlich mit der berüchtigten Novelle Wälsungenblut, die mit ebendiesem Großbürgertum noch einmal scharf ins Gericht geht, zugleich aber autobiographisch die Vorgeschichte jener Heirat erzählt.

Im so definierten Frühwerk kommt kaum ein Text ohne jüdische Figuren aus. Die Art und Weise aber ihres frühen Vorkommens sollte man sich vergegenwärtigen, um den sehr beträchtlichen Fortschritt ermessen zu können, den die späteren Texte in dieser Hinsicht bedeuten. Die jüdischen Charaktere des Frühwerks sind nach einem und demselben Muster gestrickt. Juden erscheinen hier regelmäßig als gesellschaftliche Konkurrenten und sexuelle Rivalen 'deutscher' Figuren, die ihnen ebenso regelmäßig unterlegen sind. Im allerersten erhaltenen und publizierten Erzähltext des Autors, der Novelle Gefallen, unterliegt ein blonder Enthusiast und Weltverbesserer namens Laube einem zynischen "Dr. med. Selten" (8: 11) ${ }^{1}$ intellektuell insofern, als dieser ihm gegenüber in einer Herrenrunde das letzte Wort behält. Thomas Buddenbrook unterliegt in oeconomicis langsam, aber sicher Hermann Hagenström, der endlich sogar das Haus der Buddenbrooks übernimmt und dabei die Penetrationssymbolik seines Einzugs ziemlich schamlos ausspielt. ${ }^{2}$ Tonio Kröger 
unterliegt in eroticis oder homoeroticis gleich zu Anfang Erwin Jimmerthal, der erfolgreicher um die Gunst Hans Hansens buhlt als er. Anton Klöterjahn unterliegt Detlev Spinell, soweit dieser die Tristan- und Verführerrolle besetzt. Unter dem Nebenpersonal derselben Tristan-Novelle unterliegt statuell wie ökonomisch ein Doktor Müller ("überhaupt nicht der Rede wert") dem Leiter der "Anstalt," Doktor Leander, der sich ganze "Vermögen ausliefern" (8: 21618) lässt. In Gladius Dei unterliegt ein "christliche[r] Jüngling"—-so die erste erhaltene Notiz dazu ${ }^{3}$ - besonders drastisch und augenfällig dem Kunsthändler M. Blüthenzweig, der ihn mit brachialer Gewalt vor die Tür setzen lässt. Und endlich, in Wälsungenblut, unterliegt der gutmütige Beamte von Beckerath seinem Schwager in spe, Siegmund Aarenhold, der ihn sozusagen um das ius primae noctis prellt. Die Juden werden dabei nie als solche benannt. So schrieb Thomas an Heinrich Mann in einem Brief zwar nur über diese von ihm selber 'unter Brüdern' so genannte "Judengeschichte" und "TiergartenNovelle," ${ }^{5}$ Wälsungenblut, der aber die entsprechende Systematik und Intentionalität für das ganze Frühwerk mit wünschbarer Deutlichkeit dokumentiert: "Das Wort 'Jude, jüdisch' kommt nicht vor."

Indem sie "[d]as Wort" aussparen, erzeugen die frühen Texte eine ganz bestimmte Appellstruktur und machen sie eine genau bestimmbare Rezeptionsofferte. Sie bieten ihrer Leserschaft ein Ratespiel an. Das Spiel besteht darin, den jüdischen Rivalen auch unter den Bedingungen seiner nahezu vollkommenen Akkulturation zu erkennen, ihn trotz dieser Akkulturation im Wortsinn zu 'diskriminieren,' das ausgesparte "Wort" also selbständig in die vorsätzlich offen gehaltene Leerstelle einzutragen. Gewährleistet wird die Identifizierbarkeit 'des' Juden auf doppelte Weise. Zum einen hat 'er' einen stereotyp jüdischen Namen zu tragen: Spottnamen wie "Jimmerthal"; ad malam partem sprechende Namen wie "Hagenström"; Wunschnamen wie "Blüthenzweig"; Edelsteinnamen oder immerhin "event[uelle]" Edelsteinnamen wie "Spinell"; Assimilationsnamen wie "Hermann," "Detlev" oder "Siegmund"; Fluchtnamen wie "Aarenhold" oder "M."; oder sonstwie 'typische' oder fremde Namen wie "Selten" ${ }^{8}$ und "Leander." Zum anderen ist 'sein' Äußeres, wenngleich meistens nur "ein wenig," mit stereotyp 'jüdischen' Merkmalen behaftet: die Nase "ein wenig platt" (1: 64; 8: 206) ${ }^{9}$, die Augen "blank" $(8: 177)^{10}$, der Bartwuchs stark $^{11}$ oder aber fehlend ${ }^{12}$, das Haar dunkel ${ }^{13}$ und kraus. ${ }^{14}$ Das Frühwerk ist also nicht frei von antisemitischen Zügen. Oder jedenfalls war es zu seiner Zeit geeignet, an eine antisemitisch gesinnte Leserschaft zu appellieren; mag der amerikanische Exulant später, 1940, aus der Retrospektive auch behauptet haben, dass Antisemitismus damals, "thirty-five years ago," in Deutschland eine Seltenheit gewesen sei (in der apologetischen Absicht, einer entsprechenden Lektüre ausgerechnet von Wälsungenblut zuvorzukommen). ${ }^{15}$ Der frühe Thomas Mann war offensichtlich nicht ganz resistent gegen den Sog des rassistischen Antisemitismus, wie er sich im Deutschen Reich im letzten Drittel und besonders im letzten Jahrzehnt des 19. Jahrhunderts politisch aus 
einer komplexen Gemengelage heraus zu formieren begann. Zu dieser gehörte bekanntlich das Aufkommen von Evolutionsbiologie und Rassenhygiene, die rechtliche Gleichstellung der jüdischen Deutschen, deren teilweise sehr erfolgreiche Assimilation und Karriere vor allem in Handel und freien Berufen, die Verschärfung von Konkurrenz und Existenzangst während der Großen Depression. Individualpsychologisch lässt sich Thomas-und folgerichtig auch Heinrich-Manns Anfälligkeit für den in seinen-beziehungsweise ihrenformativen Jahren landläufigen Antisemitismus leicht erklären. Die Familie gehörte wie die Buddenbrooks zu einer wirtschaftlich besonders gefährdeten Gruppe des deutschen Besitzbürgertums. ${ }^{16}$ Nach dem Tod seines Vaters, nach dem Verkauf der väterlichen Firma und nach seinem Scheitern im Gymnasium musste sich Thomas Mann als Deklassierter vorkommen. Er hatte sein ökonomisches und soziales Kapital weitgehend verloren und vermochte diese Einbuße einstweilen noch nicht mit kulturellem oder symbolischem Kapitalgewinn aufzuwiegen. Solch eine psychologische Erklärung lässt sich auch $e x$ negativo erhärten. Denn mit zunehmendem Erfolg des Autors werden die im eben beschriebenen Sinn patenten Antisemitismen immer seltener, und endlich scheinen sie ganz auszubleiben. Aufschlussreich für diesen Mentalitätswechsel ist eine "Skizze" aus dem Jahr 1904, Beim Propheten. 'Der' Jude, jetzt konsequenterweise auch als solcher bezeichnet (wenn nicht geradeheraus mit dem "Wort," so immerhin mit der habitualisierten Synekdoche "Semit" [8: 362]), ist nun seinerseits einer Figur weit unterlegen, die unverkennbar die Züge und selbst die äußeren Merkmale des realen Autors trägt ${ }^{17}$, einem erfolgreichen und sehr salonfähigen "Novellist[en]" (8: 363), der eben dabei ist, sich vermöge seines Erfolgs und seiner Salonfähigkeit aufs beste zu liieren.

Im Jahr darauf, 1905, entstand dann zwar noch jene "Tiergarten-Novelle." Aber schon deren Publikationsgeschichte verrät gleichsam das schlechte Gewissen, mit dem der Autor hier nochmals ins antisemitische Register griff. Wälsungenblut erschien erst viel später, 1921, und dann zu Lebzeiten des Autors nie mehr wieder auf Deutsch, in englischer Übersetzung erst weitere anderthalb Jahrzehnte später. ${ }^{18}$ Die Auflage der Erstausgabe war sehr niedrig, auf nur 530 Exemplare limitiert. Und die Exemplare dieser Luxusausgabe waren für eine breitere Leserschaft unerschwinglich teuer. Ein Exemplar kostete, nota bene vor der Inflationszeit, bis zu 3000 Mark (während, zum Vergleich, die im Jahr darauf erschienene Ausgabe des Felix Krull für 25 Mark zu haben war). In absentia zu beobachten ist der Zusammenhang von antisemitischem Ressentiment und persönlicher Frustration zum Beispiel auch an einer öffentlichen Stellungnahme, die Thomas Mann 1907 zur "Lösung der Judenfrage" abgab und in der er sich als "Philosemit[en]" titulierte ${ }^{19}$, oder auch an dem Roman von 1909, in dem er auf der autobiographischen Ebene die Restitutionsgeschichte seiner diversen Kapitalien mit erzählte, Königliche Hoheit. Der hier auftretende Jude, Doktor Sammet, trägt zwar seinerseits einen typisch 'jüdischen' Namen (nach typischem Handelsgut) und ist nach wie vor mit einer 
stereotyp 'jüdischen' Nase geschlagen. Aber er ist nicht mehr dem vordem üblichen Ratespiel ausgeliefert und wird der rassistischen Neugier gar nicht erst ausgesetzt; sondern seine "Abstammung" wird im Text selbst thematisch (2: 14), ja selbst der Antisemitismus wird es, und zwar in Hinblick auf eine förderliche Wirkung, die er auf den Ehrgeiz und die Karrieredisziplin des Diskriminierten, wie dieser selber findet und gut findet, haben soll. Darüber hinaus ist der Kinder- und Armenarzt Sammet, wenn auch durchaus keine einnehmende Erscheinung, so doch, als erster Jude des Gesamtwerks, ein rundum sympathischer Charakter. Die Konkurrenz, die seine Befähigung für die anderen Ärzte bedeutet, scheint er nicht selbst gesucht zu haben—er wird vom Fürsten ohne eigenes Zutun berufen-; und die antisemitischen Reflexe, die er bei diesen anderen wachruft, werden in ihrer menschlich-allzumenschlichen Bedingtheit einigermaßen deutlich entlarvt.

\section{II}

Vier Jahre nach der Vollendung von Königliche Hoheit begonnen (1913) und im ersten der vier, fünf 'Goldenen Jahre' der Weimarer Republik auf denmit der Währungsreform eben erst wieder stabilisierten-Markt gekommen (1924), übergreift die Entstehung des Zauberbergs den Ersten Weltkrieg und einen 'guten' Teil der Zwischenkriegszeit. Sozialgeschichtlich ist der Roman damit in ein Umfeld gestellt, in dem sich der deutsche Antisemitismus aus den bekannten Ursachen zusehends radikalisierte: Der Krieg wurde verloren, und mit der Dolchstoßlegende und in den angeblich jüdischen Kriegsgewinnlern fand man geeignete Sündenböcke dafür. Die Wirtschaft durchlief turbulente, zum Teil stark rezessive Phasen. Und die Hyperinflation (von der die angeblich jüdischen Schieber und Kapitalisten ihrerseits wieder profitierten) brachte etliche Menschen nicht mehr nur an den Rand des Ruins. In diesem Reizklima, hochgepeitscht von der nationalsozialistischen Hetzpropaganda, gedieh ein Judenhass, der auch in seinen offen formulierten Konsequenzen sehr viel weiter ging als der Vorkriegsantisemitismus und der dessen später nahezu pauschale Leugnung durch den Exulanten wo nicht rechtfertigen, so vielleicht doch mit erklären kann. Zum Beispiel erschienen im sogenannten Krisenjahr 1923, als sich Thomas Mann an das letzte Kapitel des Zauberbergs machte, in Nürnberg schon Plakate, die dazu aufriefen, Juden "wie Hunde totzuschlagen." ${ }^{20}$ Auf der Insel Usedom, wo er den Roman vollendete, waren "in den Restaurants" "Gedichte $[\ldots]$ angeschlagen," "die von der Vernichtung der Juden handeln." 21 Gegen diesen verschärften Antisemitismus nun bezog der inzwischen auch international höchst renommierte, nämlich mit dem Nobelpreis ausgezeichnete Autor öffentlich Stellung. So gehörte "Professor Dr. h.c. Thomas Mann" 1930, zusammen etwa mit Gerhart Hauptmann, zu den Unterzeichnern eines "[a]n das deutsche Volk!" gerichteten Manifests “[g]egen die Kulturschande des Antisemitismus" (um freilich noch dem in dieser Hinsicht nach dem Dafürhalten 
der Unterzeichneten respektablen "italienischen Ministerpräsidenten Mussolini" die Reverenz zu erweisen). ${ }^{22}$ Dass auch der Zauberberg, zumindest oder besonders zu seiner deutlich größeren, seiner nach dem Ersten Weltkrieg geschriebenen Hälfte, in eine Zeit gehört, in der Thomas Mann seine frühen Antisemitismen hinter sich gelassen und sich als entschiedener Gegner des völkischen Rassismus profiliert hatte, ist dem Roman prima vista anzusehen. Hans Castorps Rivale, Mynheer Peeperkorn, von dem auch unentscheidbar bleibt, ob er in der Konkurrenz um die gemeinsam begehrte Clawdia Chauchat gewinnt oder unterliegt, ist kein Jude, sondern Germane, Niederländer (oder jedenfalls Kolonial-Holländer und damit doch nur von "leicht farbige[r] Nationalität" [3: 758]). "Das Wort 'Jude, jüdisch' kommt" gegebenen Orts durchaus "vor," insgesamt (das heißt mit allen Ableitungen und Komposita) vierzehnmal: am häufigsten im Zusammenhang mit Leo Naphta, am zweithäufigsten in einem Kontext, in dem der Antisemitismus ausdrücklich thematisch wird, und zwar ganz anders und viel prononcierter als in der Verharmlosung und Beschönigung noch von Königliche Hoheit. Der Antisemitismus wird hier sogar zum Gegenstand einer Satire, nota bene zum einzigen Mal in Thomas Manns Erzählwerk (wenn man einmal von den "antisemitische[n] Äußerungen" eines deutschen Schmarotzers absieht, der im Doktor Faustus von der Großzügigkeit "jüdische[r] Verlegersfrauen und Bankiersdamen" profitiert [6: 227]). Diese Satire findet sich im zweitletzten Unterkapitel des siebten und letzten Großkapitels, "Die große Gereiztheit"; in der erzählten Zeit heißt das unmittelbar vor Ausbruch des Kriegs und entstehungsgeschichtlich zu einer Zeit (wahrscheinlich unmittelbar nach jenem 'Krisenjahr' ${ }^{23}$ ), als der nationalsozialistische, wie man ihn damals nannte, 'Radauantisemitismus' einen ersten Höhepunkt durchlief. "Die große Gereiztheit," eine ihrerseits nachgerade infektiöse oder epidemische Aggressivität der Tuberkulosepatienten, wird in fünf regelmäßig wachsenden Episoden vorgeführt, deren dritte und mittlere eben die Satire auf den deutschen - jedenfalls von einem Deutschen repräsentierten-Antisemitismus bildet: nach dem Streit einer Patientin mit einer Verkäuferin des Kurorts und dem jähzornigen Ausfall eines halbwüchsigen Patienten gegen eine Angestellte des Kurhauses, die "Zwergin" (3: 949) Emerentia; vor einem "polnische[n] Ehrenhandel" (3: 956) und endlich den zusehends aggressiveren Auseinandersetzungen zwischen Lodovico Settembrini und Leo Naphta. Während die "polnische Angelegenheit" "dem durch das Ehrenrecht angezeigten Wege" zur Satisfaktion nur ein kurzes Stück folgt und lediglich mit einem "Schnellfeuer offizieller Ohrfeigen" endet (3: 952-57), führen Naphtas und Settembrinis intellektuelle Auseinandersetzungen zuletzt zu einem Pistolenduell. Dabei fällt Naphta, wenngleich auf "eine Weise, die nichts mehr mit Kampf zu tun hat[]" (3: 980). Das Duell, das "mit Kampf" nichts "zu tun hat[]," aber in dem der jüdische Kontrahent dennoch, wenn auch 'nur' durch seine eigene Hand umkommt, wird in der mittleren Episode, im Streit zwischen dem Antisemiten Wiedemann und dem Juden Sonnenschein 
gleichsam präludiert, der einzigen weiteren "Gereiztheit," bei der es ebenfalls um einen Streit 'von Mann zu Mann' geht (und nicht um einen zwischen Frau und Frau, Mann und Frau beziehungsweise Jüngling und Zwergin oder, wie im "Ehrenhandel," zwischen ganzen Gruppen). In dem brachialen Zweikampf, bei dem Wiedemann und Sonnenschein zu "kleine[n] Jungen" regredieren und der sich gleichwohl im Vergleich mit jenem lächerlichen "Ehrentrubel" noch als "Wohltat" erweisen soll (3: 952), wird die als besonders kultiviert geltende Aggressionsform des Waffenduells virtuell auf ein vormenschliches Niveau abgesenkt:

Sie katzbalgten sich wie kleine Jungen, aber mit der Verzweiflung erwachsener Männer, mit denen es dahin gekommen ist. Sie gingen einander mit den Krallen ins Gesicht, hielten sich an Nase und Kehle, während sie aufeinander losschlugen, umschlangen sich, wälzten sich in furchtbarem und radikalem Ernste am Boden, spien nacheinander, traten, stießen, zerrten, hieben und schäumten. [ ... ] Wiedemann, speichelnd und blutend, wutverblödeten Angesichts, zeigte das Phänomen der zu Berge stehenden Haare. [ . . . ] Die Haare standen Herrn Wiedemann starr und steif zu Berge, und so stürzte er davon, während Herr Sonnenschein, das eine Auge in Bläue verschwunden und eine blutende Lücke in dem Kranz lockigen schwarzen Haares, das seinen Schädel umgab, [ . . . ] sich niederließ und bitterlich in seine Hände weinte. (3: 951 f.)

Die beiden auf so "tierische Weise" (3: 951) ineinander "Verbissenen und Verkrallten" (3: 951) machen eine vielleicht gleichermaßen unwürdige, aber doch verschieden erbärmliche Figur. Wie in jenem mörderischen oder selbstmörderischen Duell, das dadurch aufhört, eines zu sein, der Jude Naphta weniger 'fällt' als einfach liegenbleibt, so lässt auch hier der Jude Sonnenschein mehr oder 'lässt' eigentlich nur er 'Haare' (während Wiedemanns "steif" "stehende[s]" Haar im Gegenteil an Volumen zulegt). Er bleibt in der Haltung des Geschlagenen und 'Kastrierten' zurück. Und obendrein scheint er, von fern wenigstens, nämlich über eine etwas irritierende Evangelienreminiszenz, an einen Juden zu erinnern, der seinen "Herrn" verleugnete ("bitterlich $[\ldots]$ weinte"). ${ }^{24}$

Ursache dieses Kampfes, der also doch eher zu Ungunsten des Juden ausgeht, sind die antisemitischen "Sticheleien" (3: 951) Wiedemanns. Dessen Antisemitismus, als "Stolz und Inhalt seines Lebens," durchschaut der Erzähler von allem Anfang an als Komplement eines sozialen und gesundheitlichen Ruins:

Ein Mann trat in die Berghofgemeinschaft ein, ein ehemaliger Kaufmann, dreißigjährig, schon lange febril, seit Jahren von Anstalt zu Anstalt gewandert. Der Mann war Judengegner, Antisemit, war es grundsätzlich und sportsmäßig, mit freudiger Versessenheit,- - die aufgelesene Verneinung war Stolz und Inhalt seines Lebens. Er war ein Kaufmann gewesen, er war es nicht mehr, er war nichts in der Welt, aber ein Judenfeind war er geblieben. Er war sehr ernstlich krank, hustete schwer beladen und tat zwischendurch, als ob er mit der Lunge nieste, hoch, kurz, einmalig, unheimlich. Jedoch war er kein Jude, und das war das Positive an ihm. Sein 
Name war Wiedemann, ein christlicher Name, kein unreiner. Er hielt sich eine Zeitschrift, genannt 'Die arische Leuchte,' und führte Reden wie diese:

"Ich komme ins Sanatorium X. in A.... Wie ich mich in der Liegehalle installieren will,- -wer liegt links von mir im Stuhl? Der Herr Hirsch! Wer liegt rechts? Der Herr Wolf! Selbstverständlich bin ich sofort gereist" und so weiter.

'Du hast es nötig!' dachte Hans Castorp mit Abneigung.

[ . . . ] Die Mißidee, die ihn [Wiedemann] ritt, war zu einem juckenden Mißtrauen, einer rastlosen Verfolgungsmanie geworden, die ihn trieb, Unreinheit, die sich in seiner Nähe versteckt oder verlarvt halten mochte, hervorzuziehen und der Schande zuzuführen. Er stichelte, verdächtigte und geiferte, wo er ging und stand. Und kurz, das Betreiben der Anprangerung alles Lebens, das nicht den Vorzug besaß, der sein einziger war, füllte seine Tage aus. (3: 950)

Was der Erzähler hier psychologisch analysiert und in eigener Instanz wie auch Hans Castorp unmissverständlich verurteilt, ist im Grunde nichts oder jedenfalls nichts wesentlich anderes als das Versteck- und Demaskierungsspiel, zu dem die gewissen Appellstrukturen in Thomas Manns eigenem Frühwerk dessen Leser anstifteten. Ginge es vielleicht auch etwas zu weit, den Text deswegen gleich als Selbstabrechnung des Autors mit diesem Aspekt seiner Vorgeschichte zu lesen, so bleibt doch einigermaßen bemerkenswert, dass der entsprechende Zug an Wiedemanns "Mißidee" hier pragmatisch gesehen eigentlich vollkommen entbehrlich wäre. Seine Obsession, die in seiner Nähe "versteckt[en] und verlarvt[en]" Juden "hervorzuziehen," kann hier gar nicht erst ein- und ansetzten:

[E]s war da ein anderer Mann,-_zu entlarven gab es nichts, was ihn betraf, der Fall war klar. Dieser Mann hieß Sonnenschein, und da man nicht schmutziger heißen konnte, so bildete Sonnenscheins Person vom ersten Tag an die Puschel, die vor Wiedemanns Nase hing [ . . . ]. (3: 951)

Die "Verfolgungsmanie," assimilationswillige Juden an ihren "schmutzige[n]" Namen zu erkennen (zum Beispiel Wunschnamen wie "Sonnenschein" oder Tiernamen wie "Hirsch" oder "Wolf") oder sie aber an ihren nicht assimilierbaren Körpermerkmalen zu identifizieren (Sonnenschein zum Beispiel an "lockigen schwarzen Haare[n]"), kann hier also dadurch parodiert werden, dass sie auf die romaninterne Handlung verlagert beziehungsweise in eine Figurenrede verschoben wird. In direkte "Reden" verschoben ist die Identifikation auch des vielleicht berüchtigtsten und nach Nathan dem Weisen überhaupt berühmtesten Juden der deutschen Literatur, Leo Naphtas eben, den Thomas Mann nach dem 'Vorbild' eines als solcher bekannten Juden formte, Georg Lukács' (woran der Name von Naphtas und Settembrinis Vermieter gleichsam metonymisch erinnert: der Damenschneider Lukaček "mit stark böhmischem Akzent," der schon mit seinem allerersten Wort, "Grütsi," weil es "weder zu seinem Namen noch zu seinem Äußeren paßt[] und etwas falsch und sonderbar" sich ausnimmt, eine im "Schweizerische[n]" eh und je eher 
ungern gesehene Assimilationsfreudigkeit an den Tag legt [3: 543]). Dieser prominenteste oder einzig prominente Jude des Romans tritt seinerseits erst in dessen nach dem Krieg geschriebener Hälfte auf, nachdem die Gegenposition zu Settembrini ursprünglich ein evangelischer Pastor namens Bunge hätte besetzen sollen, das muss wohl heißen eine sowohl religiös als auch ethnisch denkbar unverdächtige Gestalt. Der terminus post quem dieser Umbesetzung fällt in den Januar 1922, als Thomas Mann Georg Lukács persönlich kennenlernte; der terminus ante quem in den Juni desselben Jahrs, weil zu diesem Datum in der Korrespondenz erstmals ein Leo Naphta "aufgetaucht" ist: "ein" hier erst "halbjüdischer Jesuitenzögling mit krassen Anschauungen" und "beständig mit Herrn Settembrini in scharfen Disputen [ . . . ], die eines Tages zum pädagogischen Duell führen werden." ${ }^{25}$ Naphta erscheint also enstehungschronologisch knapp zwei Jahre vor Sonnenschein, erzählzeitlich im sechsten und zweitletzten Großkapitel und in der erzählten Zeit nicht von ungefähr während der unheimlichen Phase der Sommersonnenwende. ${ }^{26}$ Dabei wird er nicht nur mit dem sehr einschlägigen Namen Leo 'Leib' Naphta belegt und mit einem ausgeprägt, nicht mehr nur "ein wenig” jüdischen Äußeren versehen:

Es stellte sich heraus, daß der Fremde, der Settembrini's Jahre haben mochte, dessen Hausgenosse war: der andere Aftermieter Lukaçeks [sic], des Damenschneiders, Naphta mit Namen, soviel die jungen Leute verstanden. Er war ein kleiner, magerer Mann, rasiert und von so scharfer, man möchte sagen: ätzender Häßlichkeit, daß die Vettern sich geradezu wunderten. Alles war scharf an ihm: die gebogene Nase, die sein Gesicht beherrschte, der schmal zusammengenommene Mund, die dickgeschliffenen Gläser der im übrigen leichtgebauten Brille, die er vor seinen hellgrauen Augen trug, und selbst das Schweigen, das er bewahrte und dem zu entnehmen war, daß seine Rede scharf und folgerecht sein werde. [ . . . ] Settembrini's [ . . . ] Genosse, obgleich blonden Haares-es war übrigens aschblond, metallisch-farblos, und er trug es glatt aus der fliehenden Stirn über den ganzen Kopf zurückgestrichen-, zeigte gleichfalls die mattweiße Gesichtshaut brünetter Rassen. [ . . . ] Naphta legte nach erfolgter Vorstellung sogleich wieder die Hände auf dem Rücken zusammen. Sie waren klein und zart, wie auch seine Füße sehr zierlich waren, übrigens seiner Figur entsprechend. Daß er erkältet wirkte und auf eine gewisse schwächliche und unförderliche Art hustete, fiel nicht auf. (3: 517 f.)

“[K]lein[], mager[]," “ätzende[] Häßlichkeit," eine "gebogene Nase" und "Brillengläser[]" so "dick[]" wie diejenigen Doktor Leanders . . . (8: 216): Die hier ganz eindeutigen Markierungen, die einen antisemitischen Spürgeist ohnehin so wenig herauszufordern vermöchten wie die Gestalt oder der Name Sonnenscheins ${ }^{27}$, werden an- und abschließend in Figurenrede auf den Nenner des "Wort[s]" wie dann auch noch jener habituellen Synekdoche des 'Semitischen' gebracht. Auf die Frage: "Wie hat er dir gefallen?" lehnt Joachim Ziemßen die Person Naphtas kategorisch ab, nicht etwa dessen ideologische 
Positionen, die er teils ausdrücklich gutheißt. Er argumentiert dabei indessen nicht eigentlich ad hominem, sondern ad gentem:

"Der Kleine? Nicht gut. Er sagte manches, was mir gefiel. [ . . . ] Aber er selbst hat mir wenig gefallen, und da kann einer noch soviel Gutes sagen, was habe ich davon, wenn er selbst ein zweifelhafter Kerl ist. Und zweifelhaft ist er, das kannst du nicht leugnen. [ . . . ] Und dabei hat er ja eine Judennase, sieh ihn dir doch an! So mickrig von Figur sind auch immer nur die Semiten. [ . . . ]" (3: 534)

Dass "[d]er Kleine" “[n]icht gut" und "wenig" gefällt, hat also primär mit seiner Nase und seinem Körper zu tun und ist damit wieder Teil und Ausdruck des deutschen Vorkriegsantisemitismus, den der Exulant Thomas Mann später fast ganz in Abrede stellen sollte. Die Figur, die diese schneidige Verachtung alles 'Semitischen' artikuliert, ist keine beliebige und schon gar keine vom Typus des ruinierten, frustrierten und von schlimmen Minderwertigkeitsgefühlen gequälten Jammermenschen Wiedemann. Artikulieren lässt der Autor diesen preußisch-militaristischen Antisemitismus, und das müsste eigentlich bereits sehr zu denken geben, eine Figur, die einen typisch märkischen und als solcher schon von Fontane her stark positiv besetzten Vornamen trägt ${ }^{28}$ und auf die er, diesem Namen und seiner Besetzung genau entsprechend, die Lesersympathie bisher geradezu konzentriert hat. Ganz anders als bei der jüngeren Witzfigur Wiedemann erhält Ziemßens antisemitische Gesinnung keine ironischen oder gar satirischen Glanzlichter aufgesetzt. Sie kann der rundum positiven Besetzung des "brav[en]" (3: 290, $702,739,750,772)^{29}$ Vetters nicht das Geringste anhaben. Und noch dazu wird der auch hier an eine Figur delegierte Antisemitismus von auktorialer Seite gleichsam ratifiziert: durch eine extrem unsympathische Gestaltung Naphtas, nicht nur seines "ätzend[]" hässlichen Äußeren, sondern vor allem auch seines terroristischen und blutrünstigen Fanatismus; implizit vielleicht auch durch die poetische 'Gerechtigkeit,' dass Naphta, und zwar auf besonders groteske und unwürdige Art, im Duell umkommt (wie ja auch schon bei dessen rein brachialer und deswegen harmloserer Antizipation 'der' Jude die schlechtere Figur macht). Den Widerspruch zwischen einem erst, bei ZiemBen, für Autor und intendierte Leserschaft offensichtlich goutierbaren oder gar respektablen, dann, bei Wiedemann, ebenso offensichtlich bösartigen und lachhaften Antisemitismus kann man schwerlich oder jedenfalls nicht restlos auflösen (etwa über die verschiedenen, aber zeitgeschichtlich und biographisch kaum weit genug auseinander liegenden Entstehungszeiten der betreffenden Kapitel). Eher hat man ihn wohl stehenzulassen und als Symptom zu begreifen. Symptomatisch scheint er dafür zu sein, dass auch ein Thomas Mann sich nicht ohne weiteres und nicht gänzlich vom eingefleischten Antisemitismus seines formativen Milieus loszumachen vermochte. Dieser scheint in den späteren Texten noch unterschwellig herumzugeistern. 
Darauf deuten auf einen zweiten Blick zum Beispiel auch vereinzelte, allerdings, wohl bezeichnenderweise, sehr randständige Figuren desselben Zauberberg-Romans. Solche Figuren (auch ihre, gemessen an den realen demographischen Verhältnissen, stark überproportionale Häufung) ließen sich durchaus noch in die einschlägigen Schemata des Frühwerks integrieren. Sie provozieren ex nomine oder obendrein noch über Herkunftsangaben, Körpermerkmale oder Charaktereigenschaften die Leserschaft dazu, das in ihren Portraits je ausgesparte "Wort" in eine offen gehaltene Leerstelle selber einzusetzen. Sie stehen damit zu 'klaren' beziehungsweise explizit klargestellten 'Fällen' wie Sonnenschein, Naphta oder der rundheraus als "rumänische[] Jüdin" bezeichneten, "schlecht und recht Frau Landauer" (3: 758) heißenden Geliebten einer ägyptischen Prinzessin in demselben Abstand, der zwischen den widersprüchlichen auktorialen Wertungen von Ziemßens und von Wiedemanns Antisemitismus liegt.

Eine "dicke Frau Salomon aus Amsterdam" (3: 108) zum Beispiel, nach einer erst der maliziösen "Klatschsucht" einer besonders missgünstigen Mitpatientin zugeschriebenen Behauptung, die der Erzähler dann aber später in eigener Instanz und ohne Vorbehalt wiederholt und damit beglaubigt (3: 499), trägt "zur Untersuchung" "die kostbarste Spitzenwäsche," um sich jeweils "vor den Ärzten mit feinem Unterzeug" zu "ziere[n]" (3: 416). Sie bedient damit eine offenbar sehr zeitgemäße Phantasie, wie sie sich aus der popular culture erschließen lässt. Denn die aufreizende Unterbekleidung der Jüdinnen gehörte ins stabile Motivarsenal wilhelminischer Postkarten..$^{30}$ Als weitere Figuren dieses Portraittyps wären etwa eigens noch zu untersuchen: Dr. Leo Blumenkohl aus Odessa oder Professor Kafka mit seinem perfiden und unredlichen "Erwerbssinn" (3: 91); "Hermine Kleefeld [ . . ] mit dummen Augen" (3: 99), "[d]ie elfenbeinfarbene Levi" (3: 73, 99, 177, 503, 932), die "fette[] und leberfleckige[] Iltis" (3: 73, 99), die "wollhaarige[] Tamara" (3: 293, 455). ${ }^{31}$ Daneben aber, und das ist für die hier einschlägige Ambiguität des Romans ebenso bezeichnend wie der Widerspruch zwischen völliger Aufgabe und unterschwelliger Fortschreibung jener antisemitischen Appellstrukturen, gibt es im Zauberberg auch solche Figuren, bei denen ein oder das andere Merkmal eine jüdische Identität zu suggerieren vermag, die aber doch in dieser Hinsicht zu vage bleiben, als dass das Identifikationsspiel des Frühwerks wirklich an ihnen durchexerziert werden könnte. Zu diesen Figuren zählen zum Beispiel ein Papierfabrikantensohn, Fritz Rotbein, oder ein verklemmter und unterwürfiger "Mannheimer," "Wehsal mit Namen, Ferdinand Wehsal und"-wie Sonnenschein- "Kaufmann seines Zeichens" (3: 590); ein zusammen mit jenem Professor Kafka erwähnter Arzt von immerhin halbwegs signifikantem Namen ${ }^{32}$, Doktor Salzmann (3: 91), der seine Patienten regelrecht 'abfüllt' (wobei ausgiebiger Alkoholkonsum als Tuberkulosetherapie tatsächlich belegbar wäre ${ }^{33}$ ); ganz besonders aber ein nun wieder ziemlich nahe am Zentrum des Romanpersonals stehender Arzt: 
Dr. Krokowski begrüßte den neuen Hausgenossen mit einer gewissen heiteren, stämmigen und aufmunternden Herzhaftigkeit, als wollte er andeuten, daß Aug in Auge mit ihm jede Befangenheit überflüssig und einzig fröhliches Vertrauen am Platze sei. Er war ungefähr fünfunddreißig Jahre alt, breitschultrig, fett, bedeutend kleiner als die beiden, die vor ihm standen, so daß er den Kopf schräg zurücklegen mußte, um ihnen ins Gesicht zu sehen,- - und außerordentlich bleich, von durchscheinender, ja phosphoreszierender Blässe, die noch gehoben wurde durch die dunkle Glut seiner Augen, die Schwärze seiner Brauen und seines ziemlich langen, in zwei Spitzen auslaufenden Vollbartes, der bereits ein paar weiße Fäden zeigte. Er trug einen schwarzen, zweireihigen, schon etwas abgenutzten Sakkoanzug, schwarze, durchbrochene, sandalenartige Halbschuhe zu dicken, grauwollenen Socken und einen weich überfallenden Halskragen, wie Hans Castorp ihn bis dahin nur bei einem Photographen in Danzig gesehen hatte und welcher der Erscheinung Dr. Krokowski's in der Tat ein ateliermäßiges Gepräge verlieh. Herzlich lächelnd, so daß in seinem Barte die gelblichen Zähne sichtbar wurden, schüttelte er dem jungen Manne die Hand, indem er mit baritonaler Stimme und etwas fremdländisch schleppenden Akzenten sagte: [... ]. (3: $28 \mathrm{f}$.

Dr. Krokowski weist zwar etliche Merkmale auf, die es nahelegen könnten, auch unter ihnen 'den' assimilierten beziehungsweise eben nicht ohne Rest assimilierbaren Juden "hervorzuziehen": Erstens ist er Mediziner, wie seinerzeit überproportional viele Juden im Deutschen Reich und bei Thomas Mann; im Deutschen Reich etwa zehn bis zwanzig Prozent der praktizierenden Ärzte-bei etwa einem Prozent Anteil an der Gesamtbevölkerung-,${ }^{34}$ bei Thomas Manns, der damit diese soziale Realität also nicht einfach nur 'widerspiegelt,' zunächst gut und gern die Hälfte (nämlich bis zu Dres. Erasmi und Zimbalist im Doktor Faustus). Krokowskis Spezialgebiet, zweitens, ist mit der Psychoanalyse eine typisch 'jüdische' Disziplin (deren seinerzeit profilierte Vertreter so gut wie alle - nämlich abgesehen nur von Carl Gustav Jungtatsächlich jüdischer Herkunft waren). Sein Deutsch ist, drittens, grammatisch korrekt, aber mit, wie gehabt: "etwas fremdländisch schleppenden Akzenten" und, vielleicht bezeichnenderweise ${ }^{35}$, "mit baritonaler Stimme" gesprochen. Die Alterität, die diese gleichsam physisch behinderten "Akzente[]" verraten, legt sein Geschlechtsname, viertens, auf Osteuropa und Polen beziehungsweise sogar auf das kaschubische Dorf 'Krokowa' fest. Denn der Name ist ja ohne viel weiteres auf seine Bedeutung hin durchsichtig und seine Endung typisch polnisch. (Als solche war sie seinerzeit nachweislich negativ besetzt: "[E]s hat einen Haken, wo kowsky aufhören," steht in Irmgard Keuns nur wenig jüngerem Roman vom Kunstseidenen Mädchen. ${ }^{36}$ )

Krokowskis ausgefallener und outriert skandinavisch klingender Vorname dagegen, den der Erzähler nur ein einziges Mal benutzt und sehr spät erst preisgibt, nämlich im Incipit des Unterkapitels "Fragwürdigstes" (3: 907), 
und auf den allein dadurch schon eine gewisse Emphase zu liegen kommt,der Vorname "Edhin" also, der so (mit h) gar nicht belegbar zu sein scheint ${ }^{37}$, weist zwar nach Nordwesteuropa und somit von allem 'Semitischen' denkbar weit weg. Aber als sozusagen hypergermanischer kann er doch, fünftens, auch wieder einen übertriebenen Assimilationseifer zu erkennen geben, wie bei einem Hermann Hagenström, Detlev Spinell oder Siegmund Aarenhold. So aufgefasst, müsste er genau das wieder verraten, was er gegebenenfalls eigentlich verdecken sollte.

Auch das Äußere Krokowskis ist, sechstens, im Sinn einer solchen 'Hermeneutik des Verdachts' ${ }^{38}$ lesbar und bedeutsam: "[B]edeutend kleiner als die beiden" deutschen Vettern Castorp und Ziemßen, hat er auffallend "dunkle" Augen, schwarze Brauen und einen "ziemlich langen" schwarzen Vollbart, der wiederum wie bei Doktor Leander "in zwei Spitzen" ausläuft und "in" dem "gelbliche[] Zähne sichtbar" werden (3: 29) ${ }^{39}$, wenn er lächelt und seine Körperoberfläche sich auftut. Diese ist allerdings auch sonst gleichsam "porös[]" (8: 223) wie Detlev Spinells Gesichtshaut: "[A]ußerordentlich bleich, von durchscheinender, ja phosphoreszierender Blässe," trägt er auch noch einen "etwas" fadenscheinigen Anzug, "durchbrochene, sandalenartige Halbschuhe [...] und einen weich überfallenden Halskragen." Dieser verleiht "der Erscheinung Dr. Krokowski's" obendrein "ein ateliermäßiges Gepräge," was immer das genau heißen soll: etwas Bohemienhaft-Künstlerisches oder etwas Unechtes und Inauthentisches, Dissimulatorisches und eben bloß oberflächlich Assimiliertes.

All diese und etliche andere Merkmale-zum Beispiel der an psychische Gewalt und blanken Sadismus grenzende Druck, unter den er in jenem Unterkapitel "Fragwürdigstes," während spiritistischer Sitzungen, eine Germanin und 'echte' Skandinavierin, die junge Dänin Ellen Brand setzt—sind reflektiert oder gebündelt in der Antipathie, die Castorp der Person Krokowskis gegenüber hegt, ebenso wie auch wieder Ziemßen oder der seinerseits besonders liebenswürdige Kontrahent Naphtas, Settembrini (der Krokowskis "einen Gedanken" mit dem selben Adjektiv versieht, das in Wiedemanns erlebten Reden mit 'jüdisch' synonym ist: "schmutzig" [3: 92]). Sie reichen aber auch einschließlich solcher Antipathien bei weitem nicht aus, eine solide Appellstruktur hervorzubringen, die es zwingend nahe legte, Krokowski eigenmächtig mit dem "Wort 'Jude, jüdisch"” zu bedenken.

Die seltsame Unbestimmtheit, Unbestimmbarkeit der Figur (die wiederum dem ihr paarweise zugeordneten Arzt, im Unterschied nun zu einem Doktor Leander, institutionell oder auch physisch unterlegen ist, dem sehr entschieden 'deutschen' und "drei Köpfe höher[en]" [3: 68] Hofrat Behrens) lässt sich in diesem Fall wahrscheinlich historisch-kritisch erklären. Krokowski ist so etwas wie eine Erinnerung an einen Juden. Seine jetzige Figuration scheint gleichsam ein Fossil der früheren, nach und nach abgeschwächten Tendenz mit einzulagern, jüdische Gestalten mit einer gewissen Häme bloßzustellen 
und sie so den antisemitischen Ressentiments der Leserschaft auszusetzen. Den dafür wichtigsten Hinweis gibt die Namengebung.

Der doppelt auffällige Name "Edhin Krokowski" taucht zuerst in einem Notizbuch Thomas Manns auf. Den terminus post quem der betreffenden Notiz kann man auf Januar 1910 festlegen. Denn diese, wie gleich noch zu zeigen, lehnt sich an den Wortlaut eines im Januar 1910 erschienenen Pasquills an: "Edhin Krokowski aus Linde bei Pinne, Provinz Posen," nachträglich überschrieben mit dem unterstrichenen Vermerk "Elender." ${ }^{40}$ Die nachträgliche Überschrift deutet darauf hin, dass Thomas Mann den Namen zunächst vermutlich, wie so oft, aufs Geratewohl notiert hatte und ihn erst später für ein konkretes Projekt vormerkte. Er bestimmte ihn vorerst für eine der "Novellen," "die" er in einem Katalog der nächsten Notizbuchseite "zu machen" sich vornahm. Den betreffenden Titel setzte er dort übrigens unmittelbar vor den Arbeitstitel des Projekts, bei dessen Ausführung der Name "Edhin Krokowski" dann seine endliche Verwendung finden sollte: "[ . . . ] Ein Elender / Der verzauberte Berg [ . . . . ${ }^{* 11}$

Während also das Novellenprojekt Der verzauberte Berg sich zu einem Roman von fast sprichwörtlichem Umfang auswachsen sollte, gedieh der Elende nicht weiter. Auf diesen bezieht sich nur noch eine Notiz, die jener ersten unmittelbar folgt und deren terminus post quem wegen der darin aufgeführten Zeitschriftennummer in den September 1912 fallen muss: "Zum 'Elenden': S. Kerr über W. Rathenau, 'Pan' II No 44." ${ }^{42}$

Die Vollendung des Elenden, wie so manchen anderen steckengebliebenen Projekts auch, delegierte der Autor im Tod in Venedig an Gustav von Aschenbach, in Gestalt "jener starken Erzählung, die 'Ein Elender' überschrieben ist" (8: 450). Aus der Paraphrase, der "die berühmte Erzählung vom 'Elenden" "dort gewürdigt wird, geht hervor, wovon ungefähr die Skizze gebliebene Novelle hätte handeln sollen: von einem "weichen und albernen Halbschurken, der [ . . ] sein Weib [ . . ] ] in die Arme eines Unbärtigen treibt" (8: 455) — genau so, wie man es seinerzeit über den Verfasser jenes Pasquills herumbot, Theodor Lessing. Lessing hatte darin, unter dem Titel Samuel zieht die Bilanz $z^{43}$, Thomas Manns Entdecker und Protektor, den Kritiker Samuel Lublinski geschmäht, dann auch Thomas Mann selber, und zwar in einer Broschüre Samuel zieht die Bilanz und Tomi melkt die Moralkuh oder Zweier Könige Sturz [...], in der er eine geschönte Fassung seiner ersten Schmähschrift mit den-ihrerseits manipulierten—Dokumenten der durch sie provozierten Kontroverse und Schlammschlacht zusammenstellte. ${ }^{44}$

Thomas Mann hatte den Elenden also erst, so scheint es, als literarische Abrechnung mit Theodor Lessing konzipiert. Dessen literarisches Portrait gedachte er darin dann offenbar, wie schon der Verweis "S. Kerr" und einige andere Zeugnisse zu verstehen geben ${ }^{45}$, mit Zügen des damaligen Literaturpapsts Alfred Kerr zu kontaminieren, der zuletzt—ausgerechnet—den Tod in Venedig und die Uraufführung des Fiorenza-Dramas scharf kritisiert ${ }^{46}$, sich 
aber auch schon über dieses selbst oder über den ersten Roman "Thomas Bodenbruch[s]" mokiert hatte ("Sprach immer stolz mit Breite / Von meiner Väter Pleite"). ${ }^{47}$

Ihre Grundlage hätte solch eine literarische Figurenkontamination sicherlich in den etlichen Gemeinsamkeiten gehabt, die Kerr und Lessing in Thomas Manns Wahrnehmung teilten. Beide waren Juden. Gerade auch als solche waren sie ihm, "die Juden Kerr und Lessing," beide zutiefst verhasst; einer wie der andere, steht weiter im Tagebuch von 1918, sein "geborener Feind." ${ }^{48}$ Darüber hinaus konnten sie beide als Beispiele für ein Phänomen gelten, das pikanterweise Lessing selber Jahrzehnte später auf den Begriff bringen sollte, den 'jüdischen Selbsthass, ${ }^{49}$ und zwar in beiden Formen, die dieses Phänomen annehmen kann: der sozusagen rein reflexiven (Hass eines und desselben Juden auf sich selber) und der gleichsam transitiven (Hass eines oder mehrerer Juden auf einen anderen oder eine Gruppe anderer Juden). ${ }^{50}$

Die reflexive Variante kann man in jener sexuellen Selbsterniedrigung vermuten, wie sie Lessing nachgesagt wurde und auf die allein sich die Paraphrase des Elenden im Tod in Venedig beschränkt. Die andere Variante sah Thomas Mann nachweislich in dem Pamphlet Lessings am Werk, das in seinem Notizbuch geradewegs zitiert ist: "Edhin Krokowski aus [... ] Pinne, [ . . . ] Posen." Anzitiert sind hierin Lessings seinerzeit unmissverständlichen ${ }^{51}$ Anspielungen auf Lublinskis ostjüdische Herkunft: "Er heißt Samuel Lublinski und kommt aus Pinne in Posen." ${ }^{52}$ Solche Süffisanz wusste Thomas Mann in seiner Replik, Der Doktor Lessing, als Manifestationen jüdischen Selbsthasses oder, wie er es avant la lettre mit einem schon sehr ähnlichen Ausdruck nannte: jüdischer "Selbstverachtung" zu interpretieren. ${ }^{53}$ Und auch die zweite, auf Kerr gemünzte Notiz zum Elenden ließe sich als Beleg für Thomas Manns Sensorium für diese Form von "Selbstverachtung" verstehen. Zu "Kerr" notierte er im Umkreis des Elenden ja ebenfalls nur eines: "über W. Rathenau" und die Fundstelle dazu. Gemeint ist damit ein Artikel, den Kerr in der von ihm selber herausgegebenen Zeitschrift Pan über den von Thomas Mann sehr geschätzten Walther Rathenau veröffentlicht hatte; eine moquante Auslassung heißt das über einen seinerseits wieder als solcher bekannten und mit später tödlichen Folgen angefeindeten Juden ${ }^{54}$ (und übrigens besonders auch über dessen eigene Teilhabe an dem hier schon wieder fast auf den späteren Begriff gebrachten Phänomen: "er haßt sich" ${ }^{55}$ ).

Den Namen "Edhin Krokowski" hat Thomas Mann also aus einem Projekt übernommen, in dem er seine Aversionen gegen einen oder den anderen Juden hatte ausagieren wollen. Welche Figur so hätte heißen sollen, darüber kann man nichts Gesichertes wissen und darf man daher wohl ein wenig spekulieren. Am nächsten liegt es zunächst anzunehmen, dass die einzig notierte Namensfiktion für die wichtigste Figur, den Protagonisten reserviert war, also den 'Elenden' selbst. Der Hintergrund allerdings der "Edhin Krokowski"-Notiz zeigt genauer bedacht vielleicht in eine andere Richtung. 
Die Übereinstimmung der Ortsnamen "Pinne" und "Posen" mit Lessings Satz über "Samuel Lublinski"-wie natürlich auch die identische Namensendung_könnte es auch näher legen, "Edhin Krokowski" als Widergänger eines in Thomas Manns Augen weit weniger negativ besetzten Juden zu vermuten, Lublinskis eben, der immerhin nicht nur Lessings "Winkelpamphlet[]"56 zufolge, sondern selbst nach Thomas Manns hierin konzilianter Entgegnung darauf von stereotyp 'jüdischer' Hässlichkeit gewesen sein soll: "Herr Lublinski ist kein schöner Mann, und er ist Jude." ${ }^{57}$

Wie dem auch sei, in jedem dieser beiden Fälle wäre Dr. Krokowski im Zauberberg eine Erinnerung gleichsam an eine Figur, die offenbar so oder so eine jüdische Herkunft hätte haben und mehr oder weniger typische Merkmale hätte tragen sollen, mehr oder sehr viel weniger negativ besetzte, sei es nur ein unschönes Äußeres oder aber einen "albernen" bis verwerflichen Charakter. Und mit der besonderen Vorgeschichte der Figur wird es zu tun haben, dass Spuren solcher Merkmale mutatis mutandis am Edhin Krokowski des Zauberbergs wiederzukehren scheinen und dass man an ihm 'jüdische' Elemente zwar nicht zwingend ausmachen muss, aber doch auch, wenn man sie denn suchen will, sehr leicht finden kann. Offenbar war es Thomas Mann unmöglich, aus dem Novellenprojekt des Elenden nur eben den Namen in den Zauberberg zu übernehmen, ohne zugleich die Assoziationen hierher quasi mit einzuschleppen, die dieser Name dort einmal zusammenhielt.

Wenn sich die Figur Dr. Krokowskis also nach dem Code "Ist er Jude?" $(8: 46)^{58}$ nur vage und nicht eindeutig verorten lässt, dann scheint das auf die lange Konzeptionsgeschichte der unter diesem Namen imaginierten Figur zu deuten. Die ersten fassbaren Anfänge dieser Geschichte reichen vor den Ersten Weltkrieg zurück, in eine Epoche, in der Thomas Mann den Antisemitismus ganz offensichtlich noch nicht als "Kulturschande" wahrnahm und gegen dessen Anfechtungen nicht ganz immun war. Die eigentliche Konkretisation der Figur aber fiel dann in eine ganz andere Zeit. Der deutsche Antisemitismus einerseits hatte schon erkennbar genozidale Züge angenommen; und der inzwischen arrivierte Autor andererseits, der sich eben einer ersten Klimax seiner Erfolgsgeschichte näherte, dem Nobelpreis, konnte es sich leisten, sich über Invektiven ihm an Sozialprestige nun haushoch unterlegener Hassfiguren wie Kerr oder erst recht Lessing ohne weiteres hinwegzusetzen.

\footnotetext{
${ }^{1}$ Fortlaufende Zitate nach: Thomas Mann, Gesammelte Werke. 13 Bde. 2nd ed. (Frankfurt/ M.: Fischer, 1974).

${ }^{2}$ Vgl. Yahya Elsaghe, Die imaginäre Nation. Thomas Mann und das 'Deutsche.' (München: Fink, 2000) 157-206.

${ }^{3}$ Thomas Mann, Notizbücher. Hrsg. Hans Wysling und Yvonne Schmidlin. 2 Bde. (Frankfurt/M.: Fischer, 1991 f.) 1:182.

${ }^{4}$ Thomas Mann, Brief an Heinrich Mann. 20. November 1905. Thomas Mann. Hrsg. Hans Wysling [unter Mitwirkung von Marianne Fischer]. 3 Bde. Dichter über ihre Dichtungen 14. ([München:] Heimeran, [Frankfurt/M.:] Fischer, 1975-81) 1: 224. Vgl. 13: 472.
} 
${ }^{5}$ Thomas Mann, Brief an Heinrich Mann. 17. Oktober 1905. Thomas Mann. Hrsg. Wysling 1: 224.

${ }^{6}$ Thomas Mann, Brief an Heinrich Mann. 5. Dezember 1905. Thomas Mann. Hrsg. Wysling 1: 225.

${ }^{7}$ Mann, Notizbücher 2: 28.

${ }^{8}$ Vgl. z. B. Jüdisches Adreßbuch für Groß-Berlin. (Berlin: Goedega, 1931 [Nachdruck Berlin: Arani, 1994]) 380, s. v. 'Selten' (14 Einträge); Verzeichnis jüdischer Autoren. Vorläufige Zusammenstellung der Reichsstelle zur Förderung des deutschen Schrifttums. 6. Lieferung. ([Berlin: Masch.], 1939) 15, s. v. 'Selten' (2 Einträge); Konrad Krause, Die jüdische Namenswelt. (Essen: Essener Verlagsanstalt, 1943) 77; Heinrich W. Guggenheimer und Eva H. Guggenheimer, Jewish Family Names and Their Origins. An Etymological Dictionary. (o. O.: Ktav, 1992) 687.

${ }^{9}$ Vgl. 1: 64, 348, 409, 489, 601, 169; 8: 48, 223.

${ }^{10}$ Vgl. 1: 62, 134, 348; 8: 223.

${ }^{11}$ Vgl. 2: 28, 123; 8: 390 .

${ }^{12} \mathrm{Vgl}$. 8: 223.

${ }^{13}$ Vgl. 1: 62; 8: 12, 48, 362.

${ }^{14} \mathrm{Vgl}$. 8: 14, 216.

${ }^{15}$ Thomas Mann, Brief an Lulla Adler. 29. Oktober 1940. Thomas Mann. Hrsg. Wysling 1: 229 f.; zur Datierung vgl. Thomas Mann. Ed. Wysling 3: 603.

${ }_{16}^{16}$ Vgl. Thomas Nipperdey, Deutsche Geschichte 1866-1918. 2 Bde. (München: Beck, 1998) 1: 250-52, $391 \mathrm{f}$.

${ }^{17} \mathrm{Vgl}$. Yahya Elsaghe, Thomas Mann und die kleinen Unterschiede. Zur erzählerischen Imagination des Anderen. Literatur-Kultur-Geschlecht, Große Reihe 27. (Köln, Weimar: Böhlau, 2004) 263-65.

${ }^{18}$ Thomas Mann, "The Blood of the Walsungs." Stories of Three Decades. (London: Secker and Warburg, 1936) 297-319.

${ }^{19}$ Thomas Mann, o. T. Die Lösung der Judenfrage. Eine Rundfrage. Hrsg. Julius Moses. (Berlin, Leipzig: Modernes Verlagsbureau, 1907) 242-246: 242.

${ }^{20}$ Ludger Heid, "Der Ostjude." Antisemitismus. Vorurteile und Mythen. Hrsg. Julius H. Schoeps und Joachim Schlör. (München, Zürich: Piper, 1995) 241-251: 248.

${ }^{21}$ Katia Mann, Brief an Margarete Hauptmann. 4. August 1924, zitiert nach: Gert Heine und Paul Schommer, Thomas Mann Chronik. (Frankfurt/M.: Klostermann, 2004) 146.

${ }^{22}$ Graf Georg Arco et al., Gegen die Kulturschande des Antisemitismus. (Gerhart Hauptmann-Museum, Erkner.) Vgl. auch Graf Georg Arco et al., "Aufruf gegen den Antisemitismus." Die Friedenswarte 30.10 (1930) 312.

${ }^{23}$ Vgl. Michael Neumann, Kommentar zu: Thomas Mann, Der Zauberberg. Hrsg. Michael Neumann. Große kommentierte Frankfurter Ausgabe. Bd. 5.2. (Frankfurt/M.: Fischer, 2002) 44-46.

${ }^{24} \mathrm{Vgl}$. Lukas 22, 62; Die heiligen Schriften des Alten und Neuen Bundes deutsch von Martin Luther. 4 Bde. (München, Leipzig: Georg Müller, o. J.) 4: 159.

${ }^{25}$ Thomas Mann, Brief an Ernst Bertram. 2. Juni 1922. Thomas Mann. Hrsg. Wysling 1: 466; im Original keine Hervorhebung.

${ }^{26}$ Vgl. Franka Marquardt, "Judentum und Jesuitenorden in Thomas Manns 'Zauberberg.' Zur Funktion der 'Fehler' in der Darstellung des jüdischen Jesuiten Leib-Leo Naphta." Deutsche Vierteljahrsschrift für Literaturwissenschaft und Geistesgeschichte 81.2 (2007) 257-281: $257 \mathrm{f}$.

${ }^{27}$ Vgl. Marquardt, "Judentum und Jesuitenorden" $265 \mathrm{f}$.

${ }^{28}$ Vgl. Theodor Fontane, Der Stechlin. Hrsg. Klaus-Peter Möller. Große Brandenburger Ausgabe. Das erzählerische Werk. Bd. 17. (Berlin: Aufbau, 2001) 10.

${ }^{29} \mathrm{Vgl}$. 3: 847.

${ }^{30}$ Vgl. Helmut Gold und Georg Heuberger, hrsg., Abgestempelt. Judenfeindliche Postkarten. (Heidelberg: Umschau Braus, 1999) 205-14.

31 "Wollhaarige (Ulotriches)" standen nach der zeitgenössischen Anthropologie selbstverständlich "auf einer viel tieferen Entwicklungsstufe"; so auch bei Ernst Haeckel (Ernst Haeckel, Natürliche Schöpfungsgeschichte. [Berlin: Reimer, 1868] 514), auf dessen Autorität Oscar Hertwig seine Allgemeine Biologie basierte, eine Hauptquelle für die naturwissenschaftlichen Passagen des Zauberbergs. 
${ }^{32} \mathrm{Vgl}$ - - außer dem Junktim mit dem ungleich typischeren Namen "Kafka"-Benzion C. Kaganoff, A Dictionary of Jewish Names and Their History. (London, Henley: Routledge and Kegan Paul, 1978) 191.

${ }^{33} \mathrm{Vgl}$. Hermann Brehmer, Die Therapie der chronischen Lungenschwindsucht. 2. Aufl. (Wiesbaden: Bergmann, 1889) 272-82; P. Dettweiler und F. Penzoldt, Die Therapie der Phthisis. (Wiesbaden: Bergmann, 1887) $25 \mathrm{f}$.

${ }^{34}$ Vgl. Jacob Lesczynsky, "Statistik der Juden. Berufsstatistik." Jüdisches Lexikon. Ein enzyklopädisches Handbuch des jüdischen Wissens in vier Bänden. Hrsg. Georg Herlitz und Bruno Kirschner. (Berlin: Jüdischer Verlag, 1927 [Nachdruck Frankfurt/M.: Athenäum, 1982]) 4: 671-698: 687; Nipperdey, Deutsche Geschichte 1866-1918 1: 400; Hans-Ulrich Wehler, Deutsche Gesellschaftsgeschichte. 5 Bde. (München: Beck, 1987-2008) 4: 727.

${ }^{35} \mathrm{Vgl}$. Marc A. Weiner, Richard Wagner and the Anti-Semitic Imagination. (Lincoln: University of Nebraska Press, 1995) 177; vgl. z. B. 6: 529.

${ }^{36}$ Irmgard Keun, Das kunstseidene Mädchen. (Berlin: Universitas, 1932) 169.

${ }^{37}$ Vgl. Benno Eide Siebs, Die Personennamen der Germanen. (Niederwalluf, Wiesbaden: o. V., 1970) 134. Freundliche Auskünfte von Britta Juska-Bauer, Zürich, und Klaus Müller-Wille, Basel.

${ }^{38}$ Paul Ricœur, "Philosophische und theologische Hermeneutik." Metapher. Zur Hermeneutik religiöser Sprache. Hrsg. Paul Ricœur und Eberhard Jüngel (München: Kaiser, 1974) 24-45: 44; Hans-Georg Gadamer, "The Hermeneutics of Suspicion.” Hermeneutics: Questions and Prospects. Eds. Gary Shapiro, Alan Sica. (Amherst: University of Massachusetts, 1984) $54-65$.

${ }^{39} \mathrm{Vgl}$. Carl Röse, "Beiträge zur europäischen Rassenkunde und die Beziehung zwischen Rasse und Zahnverderbnis." Archiv für Rassen- und Gesellschaftsbiologie 2 (1905) 689-789.

${ }^{40}$ Mann, Notizbücher 2: 185.

${ }^{41}$ Mann, Notizbücher 2: 186.

${ }^{42}$ Mann, Notizbücher 2: 186.

${ }^{43}$ Theodor Lessing, "Samuel zieht die Bilanz." Schaubühne 6.3 (1910) 65-73.

${ }^{44}$ Theodor Lessing, hrsg., Samuel zieht die Bilanz und Tomi melkt die Moralkuh oder Zweier Könige Sturz. Eine Warnung für Deutsche, Satiren zu schreiben. Mit literarischen Beiträgen von Thomas Mann, Samuel Lublinski und den vierzig sittlichsten deutschen Dichtern und Denkern. (Hannover: Verlag des “Antirüpel," 1910).

${ }^{45}$ Vgl. v. a. Thomas Mann, Brief an Hans von Hülsen. 20. April 1913. Thomas Mann. Hrsg. Wysling 1: 385 f.; vgl. auch Wyslings und Schmidlins Kommentar zur Notiz "Zum 'Elenden"”: Mann, Notizbücher 2: 186, Anm. 2.

${ }^{46}$ Alfred Kerr, "Tagebuch.” Pan 3.27 (1913) 635-41; id., "Thomas Mann: Fiorenza." Das neue Drama. Die Welt im Drama III. (Berlin: Fischer, 1905) 96-100.

${ }^{47}$ Alfred Kerr, Caprichos. Strophen des Nebenstroms. (Berlin: Späth, 1926) 168.

${ }^{48}$ Thomas Mann, "Tagebucheintrag vom 5. November 1918." Tagebücher 1918-1921. Hrsg. Peter de Mendelssohn. (Frankfurt/M.: Fischer, 1979) 55.

${ }^{49}$ Theodor Lessing, Der jüdische Selbsthaß. (Berlin: Jüdischer Verlag, 1930).

${ }^{50} \mathrm{Vgl}$. Sander L. Gilman, Jüdischer Selbsthaß. Antisemitismus und die verborgene Sprache der Juden. (Frankfurt/M.: Jüdischer Verlag, 1993) 15.

${ }^{51}$ Vgl. Louis Lewin, Aus der Vergangenheit der jüdischen Gemeinde zu Pinne. (Pinne: Gundermann, 1903) 13; A. Heppner und J. Herzberg, Aus Vergangenheit und Gegenwart der Juden und der jüdischen Gemeinden in den Posener Landen nach gedruckten und ungedruckten Quellen. (Koschmin: Selbstverlag, 1909) 692.

${ }^{52}$ Lessing, "Samuel zieht die Bilanz" 66.

${ }^{53}$ Thomas Mann, "Der Doktor Lessing." Essays. Hrsg. Heinrich Detering. Große kommentierte Frankfurter Ausgabe. Bd. 14. (Frankfurt/M.: Fischer, 2002) 112-118: 116.

${ }^{54}$ Alfred Kerr, "Walther Rathenau." Pan 2.44 (1912) 1193-200.

${ }^{55}$ Kerr, "Walther Rathenau" 1198.

${ }^{56}$ Mann, "Der Doktor Lessing" 115.

${ }^{57}$ Mann, "Der Doktor Lessing" 116.

${ }^{58} \mathrm{Vgl}$. 2: 31. 